Service social

\title{
Synthèse et commentaire des écrits sur l’approche intégrée
}

\section{René Auclair}

Volume 36, numéro 2-3, 1987

Approches intégrées

URI : https://id.erudit.org/iderudit/706364ar

DOI : https://doi.org/10.7202/706364ar

Aller au sommaire du numéro

\section{Éditeur(s)}

École de service social de l'Université Laval

\section{ISSN}

1708-1734 (numérique)

Découvrir la revue

\section{Citer cet article}

Auclair, R. (1987). Synthèse et commentaire des écrits sur l'approche intégrée. Service social, 36(2-3), 286-314. https://doi.org/10.7202/706364ar

\section{Résumé de l'article}

Extrait d'une recherche sur une innovation dans la dispensation des services sociaux, cet article constitue essentiellement une recherche documentaire SUr l'approche intégrée. Les résultats sont regroupés sous deux dimensions, soit leslimites de Tapproche curative ainsi que les concepts de pratique généraliste et d'approche intégrée. d'utilisation que vous pouvez consulter en ligne.

https://apropos.erudit.org/fr/usagers/politique-dutilisation/ 
AUCLAIR, René, professeur à l'École de service social de l'Université Laval.

\section{Synthèse et commentaire des écrits sur l'approche intégrée}

\section{René Auclair}

La synthèse et le commentaire des écrits sur l'approche intégrée, qui font l'objet du présent article, sont extraits d'une recherche sur une innovation dans la dispensation des services sociaux par une équipe du Centre des services sociaux de Québec à Charlevoix. ${ }^{1}$ L'intérêt de cet article dans le cadre du thème de ce numéro repose essentiellement sur le fait que, suite à une recherche documentaire, ${ }^{2}$ nous avons recensé soixante-dix-neuf titres dont quarante-six articles de revues et trentetrois livres sur le sujet. Nous vous présentons cette synthèse selon le plan suivant :

- Les limites de l'approche curative : la critique du cas par cas ou casework, le cloisonnement des interventions, et la dépendance des populations vis-à-vis les professionnels des services.

- Pratique généraliste et approche intégrée : le développement de la pratique généraliste, l'utilisation du concept d'intégration en service social, une définition de l'approche intégrée et la présentation de différentes approches intégrées en service social.

\section{Les limites de l'approche curative}

L'organisation actuelle du réseau des services de santé et des services sociaux est à la fois spécialisée et complexe. L'implantation de ce système intégré et coordonné de services depuis la réforme des Affaires sociales (1970 à 1980) nous confronte aux limites de l'approche curative. 


\section{La critique du cas par cas ou casework}

La remise en question d'une approche ponctuelle des problèmes sociaux date déjà de plusieurs années. Un auteur américain, Harry Specht, a montré que le casework ou intervention individuelle a été l'objet de critiques virulentes dans les années soixante aux États-Unis et accusé de contribuer aux inégalités sociales et au racisme. Elle représentait, dit-il, une faillite de ce qui devait être un moyen social de prévenir la pauvreté et la discrimination.

"Le grief le plus important contre la profession a été l'orientation clinique et thérapeutique, perçue comme une façon pour la société de blâmer la victime, de placer le fardeau des problèmes sociaux sur les personnes souffrant d'inégalités, plutôt que d'éliminer les causes de cette inégalité. $n^{3}$

Alice Home, ${ }^{4}$ pour sa part, affirme que l'organisation des services consacre trop de ressources et d'énergies dans la réduction des effets individuels des problèmes sociaux au lieu de rechercher des solutions collectives. Selon elle, une telle orientation est injustifiée puisque des études scientifiques tendent à mettre en doute l'efficacité d'une approche individualisée. Même si de nouvelles approches se sont développées et sont appropriées à certains types de besoins, par exemple l'intervention à court terme et l'intervention en situation de crise, les services sociaux demeurent plus ou moins accessibles à certains clients en raison de leurs caractéristiques culturelles ou de leur éloignement géographique.

Ainsi, l'intervention sociale de cas à cas ne serait utile qu'à une minorité de personnes, car être un "bon» client du service social individualisé demande des caractéristiques et des motivations que peu de gens possèdent. Les méthodes traditionnelles d'orientation psychologique sont vues comme peu appropriées à la clientèle de milieux défavorisés où la culture privilégie une action concrète et collective plutôt qu'intellectuelle et individualisée. De plus, affirme Home, le client doit apprendre à négocier avec un système bureaucratisé et complexe avant d'obtenir une réponse à sa demande. De surcroît, les praticiens ne sont pas toujours capables d'utiliser la méthode la plus appropriée en raison d'une formation trop spécialisée.

Shaffer considère que l'approche des problèmes sociaux s'est limitée à une vision psychologique des personnes et n'a pas accordé suffisamment d'attention au changement social. Le peu d'attention à l'action sociale n'est pas étonnant, affirme l'auteur, puisque le service social nord-américain se réclame de l'idéologie libérale qui se préoccupe d'exercer un contrôle social et de maintenir le système capitaliste. II 
serait étonnant que cette profession agisse de façon contraire à ses intérêts qui sont de maintenir sa position socio-économique.

"Prenant pour acquis l'origine de classe du travail social, son engagement envers la société industrielle et sa perspective monoculaire des problèmes sociaux, qui reflète des déficits individuels plutôt que des impératifs sociaux, il ne faut pas se surprendre des développements limités en théorie et en pratique. ${ }^{5}$

Shaffer affirme que le contexte bureaucratisé de l'intervention constitue un premier obstacle mais que l'approche curative des problèmes sociaux telle que développée en service social est un deuxième obstacle qui empêche de développer une approche globale.

\section{Le cloisonnement des interventions}

D'autres auteurs ont mis en évidence le caractère partiel et fragmentaire de la pratique. Chaque mode d'intervention, en prenant comme perspective d'analyse et d'action soit la personne, soit le groupe, soit la communauté, néglige l'interrelation qui existe entre ces différentes entités. II en résulte donc une vision réduite des problèmes sociaux.

Coughlin affirme que le service social a eu tendance à considérer certains problèmes comme relevant de l'intervention individualisée, d'autres comme étant des problèmes de groupes et d'autres comme des problèmes d'organisation communautaire. Selon lui, les problèmes se trouvent ainsi définis en fonction de la méthode du praticien. ${ }^{6}$ Une telle approche comporte une double erreur : elle implique une évaluation et un traitement a priori des problèmes.

"Ceci piège le praticien dans une double erreur; il pré-évalue le problème, et il pré-détermine le traitement. Une situation humaine et, souventes fois, un problème social, sont multidimensionnels; ils sont personnels, ils sont localisés dans le contexte d'un groupe ou de différents groupes, et les deux se produisent à l'intérieur des forces sociétales et sont affectées par celles-ci. ${ }^{7}$

L'approche traditionnelle, affirme Coughlin, en effectuant une division de la réalité, en prenant comme point de départ l'une ou l'autre méthode, se trouve à nier l'interdépendance qui existe entre les personnes, le groupe, l'organisation et la communauté. En utilisant une méthode qui ne s'intéresse qu'à un seul aspect du problème, le praticien risque ainsi de ne pas identifier le véritable problème et, en conséquence, de proposer des solutions non appropriées.

Une étude de la Fédération des C.L.S.C. met en évidence les lacunes des services offerts aux 0-18 ans. On dénonce l'accent mis sur 
les "méthodes de réparation" qui nécessitent des énergies et des ressources qui seront toujours insuffisantes.

"Un tel accent sur une réponse ponctuelle aux problèmes draine des énergies et des ressources qui sont et seront toujours insuffisantes. Cette façon de procéder crée une situation où les demandes ne pourront que s'accroître, devenir de plus en plus pressantes et nécessiter la formation d'un plus grand nombre de spécialistes pour prendre en charge des problèmes de plus en plus complexes. ${ }^{8}$

Selon cette étude, une telle approche, en plus de drainer des ressources considérables à essayer de corriger et de réparer les problèmes tant physiologiques que psycho-sociaux, favorise une dépendance croissante de la population vis-à-vis les professionnels des services.

\section{La dépendance des populations vis-à-vis les professionnels des services}

L'approche centrée trop exclusivement sur la réparation ou la correction des problèmes sociaux tend à minimiser la participation des personnes et des collectivités à l'amélioration de leur bien-être. Jack Geiger a regroupé les types d'approches dans les programmes anti-pauvreté de la façon suivante :

"Ces programmes pour le pauvre, définis dans le sens classique du bien-être public; ceux par le pauvre, qui impliquent le pauvre (...) avec une participation maximale; et ces programmes du pauvre, impliquant non seulement une participation mitigée, mais aussi le contrôle et le pouvoir." ${ }^{9}$

L'approche des problèmes prend donc autant d'importance que l'action elle-même. Ainsi Davis et Tranquadea, ${ }^{10}$ à la suite d'une évaluation sociologique d'un centre de santé polyvalent, en arrivent à la conclusion que d'un point de vue strictement logique ou d'une prise de décision rationnelle, l'implantation d'un programme visant à résoudre les problèmes de santé, au niveau local, ne nécessite pas la délégation d'un pouvoir de décision : autrement dit, on devrait impliquer la communauté, mais simplement à titre consultatif. Par contre, de façon stratégique, si le succès est important, la question d'impliquer la communauté ne se pose plus. Il s'agit plutôt de se demander comment l'impliquer afin qu'elle puisse avoir suffisamment d'autonomie et de contrôle sur la gestion de ses propres affaires. ${ }^{11}$

L'implication des individus, surtout en milieux défavorisés est, selon Moynihan ${ }^{21}$, une condition nécessaire au succès de l'approche 
des problèmes. On imagine mal, en effet, comment des problèmes d'alcoolisme, de drogue ou de planification familiale pourraient se régler sans leur contribution.

L'approche des problèmes sociaux, dans cette perspective, devrait favoriser la maîtrise collective des citoyens sur leurs affaires. Elle dépasse le simple niveau de participation où la communauté est consultée mais où le pouvoir final de décision appartient à une autre instance organisationnelle ou politique.

\section{Pratique généraliste et approche intégrée}

La prise de conscience des limites d'une approche fragmentée et curative des problèmes sociaux a amené certains théoriciens à élaborer des approches qui se veulent intégrées. Celles-ci s'établissent et se définissent à l'intérieur d'une pratique généraliste et se caractérisent par une vision globale des problèmes sociaux qui accorde une plus grande importance à l'action collective.

Au plan de l'organisation des services, ces approches impliquent une participation soutenue des professionnels et des populations à la planification, à la distribution et à l'évaluation des services.

\section{Le développement de la pratique généraliste}

Selon K. Teigiser, ${ }^{13}$ l'idée d'une pratique généraliste a largement été acceptée à la fin des années 1960, comme une alternative aux approches par méthodes. À l'origine, il s'agissait d'une approche multiméthode dans laquelle une base commune était recherchée et où le travailleur social continuait de jouer des rôles traditionnels tout en augmentant le nombre de ses rôles. Cette pratique alternative recherchait donc de nouvelles conceptualisations pour une pratique caractérisée par une attention aux systèmes humains et au contexte dans lequel l'intervention avait lieu.

Garvin, ${ }^{14}$ qui a comparé différents écrits sur le sujet, estime que lorsque l'on parle de méthode intégrée, il ne ressort pas clairement s'il s'agit d'une combinaison de méthodes existantes ou bien d'une nouvelle approche de la pratique du service social. II souligne que le manque de consensus sur une définition des termes "généraliste" et "méthode intégrée " rend difficile toute comparaison de ce genre.

Pour les fins de sa recherche, Garvin a défini "pratique généraliste» comme étant les activités d'un travailleur social y incluant des interventions 
auprès des individus, des groupes et systèmes plus larges. Il parle aussi de "méthode intégrée " qu'il définit comme étant toute approche qui combine des procédures ou processus du travail social, pour intervenir auprès d'individus ou de groupes plus larges. Ceci peut se faire soit en faisant une combinaison des méthodes d'intervention individuelle, de groupe ou d'organisation communautaire ou encore en reconceptualisant la pratique.

Selon Anderson, ${ }^{15}$ le concept même de généraliste se rapporte aux connaissances et habiletés du travailleur social. Contrairement au spécialiste, le généraliste a une vaste gamme de connaissances, de méthodes et d'habiletés.

Dans la pratique généraliste, les habiletés nécessaires pour déterminer la méthode à employer sont un préalable essentiel à leur utilisation. Le cadre pour choisir ces méthodes a été élaboré à partir des écrits de Pincus et Minahan, ${ }^{16}$ qui se sont efforcés de faire la différence entre le client et le système-cible. Le client ou système-client est celui qui a fait une demande de service ou pour lequel le service est utilisé. Celui-ci est le bénéficiaire immédiat du service ou celui qui a conclu une entente de travail avec le travailleur social. Le système-cible est celui vers lequel les activités du travailleur social sont dirigées pour atteindre les buts qui ont fait l'objet d'un contrat entre les deux parties.

À l'intérieur de ces deux dimensions, les méthodes utilisées par l'intervenant peuvent être structurées à l'intérieur d'un cadre d'intervention de quatre quadrants qui s'inspire du modèle de Middleman et Goldberg. ${ }^{17}$ Les méthodes nécessaires aux services directs s'adressent :

- directement en faveur de la personne-cliente ; ici le but en est un de développement individuel et la méthode utilisée est l'intervention individuelle;

- en faveur du client et par la même occasion d'autres clients (groupes, familles...). Ici, le but visé est le développement des interrelations entre les systèmes et les méthodes retenues sont l'intervention de groupe et familiale;

- aux clients indirects en faveur du système-client. Ici, le but est de responsabiliser l'organisation; la méthode utilisée est le développement organisationnel;

- et enfin, plusieurs autres clients indirects en faveur du systèmeclient. On parle alors de développement communautaire, de développement de politiques, de défense de droits ; la méthode utilisée est donc l'organisation communautaire.

Elliot Studt ${ }^{18}$ a introduit une autre dimension de la pratique généraliste. Il parle des implications de cette pratique sur l'organisation de l'agence qui distribue les services. Elle présuppose que les intervenants 
travaillent ensemble pour trouver des solutions aux divers problèmes de leurs clientèles plutôt que de répondre aux demandes isolément. Pour ce faire, il est donc nécessaire de modifier le modèle organisationnel bureaucratique des établissements de services sociaux en faveur de plusieurs modèles qui faciliteront l'intervention des praticiens sur différents problèmes.

De plus, il doit être clair que les travailleurs sociaux ont besoin de connaissances et d'habiletés tout comme ils ont besoin d'un rôle responsable dans l'organisme qui leur permettra d'influencer la façon dont le processus de travail est établi.

Concrètement, l'organisme doit permettre aux praticiens d'avoir la possibilité d'utiliser différents modes d'intervention; de développer leurs capacités créatrices; de prendre part aux responsabilités de l'agence et d'obtenir une certaine marge d'autorité et enfin, de communiquer facilement avec les différents niveaux d'autorité.

En résumé, les méthodes traditionnelles d'intervention en service social, soit l'individuel, le groupe et le communautaire, lorsque utilisées séparément, détruisent prématurément le lien qui existe entre elles, et de plus ne favorisent pas le développement d'habiletés pour des fonctions de planification, d'administration et d'analyse de politiques.

L'approche généraliste, au contraire, exige que le praticien soit capable de composer avec des demandes de services directs et de le faire tout aussi bien pour des demandes de services indirects (planification, évaluation, organisation, analyse). L'une des critiques qui peut être apportée, selon Specht, ${ }^{19}$ c'est qu'il s'agit de demandes parfois fort exigeantes pour un même praticien.

Cette autre critique s'adresse cette fois à la profession du service social. Selon Coughlin, ${ }^{20}$ le principal problème est d'intégrer la multitude des connaissances théoriques.

\section{L'utilisation du concept d'intégration en service social}

Lowy affirme que l'intégration est l'établissement de liens entre différentes parties dans le but d'en arriver à un tout cohérent. II peut être conçu comme un état que l'on veut atteindre et aussi comme un processus qui permet l'atteinte de cet objectif.

"Perçue comme un état terminal, elle implique l'atteinte ou l'achèvement, le lien des différentes parties dans un tout utile et significatif (...). Comme processus, cependant, l'intégration réfère aux moyens utilisés pour atteindre cet état de totalité. ${ }^{21}$ 
Pour sa part, Anderson ${ }^{22}$ distingue trois niveaux d'intégration : une intégration des "connaissances", une intégration "conceptuelle" et une intégration reliée à la pratique. Le premier niveau implique l'acquisition d'informations sur l'agence-employeur et les ressources sociales, sur la base "générique " de la pratique, c'est-à-dire, le but du service social, sa fonction, la cible de l'intervention, ses valeurs et certaines habiletés. Le deuxième niveau est l'intégration "conceptuelle" qui consiste à relier les connaissances provenant de plusieurs sources différentes et à les rassembler dans un cadre cohérent. Anderson voit ce deuxième niveau comme une étape supplémentaire puisqu'elle représente un effort pour appliquer les connaissances, les valeurs et les habiletés à la pratique.

Il est donc nécessaire, selon lui, d'acquérir des principes et habiletés "génériques" en communication et sur la façon de solutionner les problèmes. Il faut ensuite évaluer les situations et effectuer le choix des méthodes d'intervention appropriées.

Le troisième niveau est l'intégration au niveau de la pratique où les connaissances et les valeurs se traduisent par un comportement professionnel. Ce niveau d'intégration prend place par [...] la traduction de ses propres connaissances et valeurs en des actions spécifiques dans un cadre de pratique. " 23 À ce niveau, affirme l'auteur, l'intégration est très personnelle : la pratique est ainsi vue comme un « art » et le praticien, un " artiste".

Selon Garvin, ${ }^{24}$ le concept d'intégration implique l'idée d'associer des méthodes. Celles-ci requièrent des connaissances, des valeurs et des modalités d'action spécifiques à chacune d'entre elles. Or, la perspective globale ou la vision holistique des situations-problèmes requiert l'établissement de liens entre différents champs de connaissances - psychologie, sociologie, science politique etc. - sur lesquels s'appuie l'intervention sociale.

En résumé, le concept d'intégration développé en service social fait référence à une approche globale des problèmes sociaux qui prend en considération les différents niveaux de facteurs qui contribuent à leur existence de même que la dynamique de leurs interrelations. Elle suppose au plan théorique, l'établissement de liens entre diverses connaissances théoriques, différentes valeurs et habiletés qui sont caractéristiques à l'ensemble des travailleurs sociaux.

Au plan de l'intervention, cette perspective multidimensionnelle requiert l'accomplissement à plusieurs niveaux d'un ensemble d'activités ou de tâches coordonnées auprès des personnes, des groupes, des organisations et des structures sociales. 


\section{Une définition de l'approche intégrée}

Pincus et Minahan ${ }^{25}$ affirment qu'il ne devrait pas exister de division du travail, c'est-à-dire d'une part offrir des services afin de répondre aux besoins individuels et d'autre part, rechercher des solutions aux enjeux collectifs. Plusieurs problèmes personnels ne peuvent être solutionnés par une personne ou une famille parce qu'ils sont reliés à des enjeux collectifs. À l'inverse, ces derniers ne peuvent être résolus sans tenir compte de leur impact sur les problèmes individuels.

L'approche intégrée que plusieurs universités américaines expérimentent veut promouvoir une vision globale, holistique des différentes responsabilités que doit assumer le travailleur social. ${ }^{26} \mathrm{Or}$, ces responsabilités impliquent des interventions cliniques (auprès d'individus, de familles ou de groupes), de même qu'auprès des organisations et des communautés.

Cette approche se définit comme plus réaliste que l'approche traditionnelle. D'ailleurs, le modèle traditionnel, affirme Evans, ${ }^{27}$ est centré sur la méthode alors que le modèle intégré est centré sur le problème.

Il se veut plus réaliste, parce qu'il est absolument nécessaire, selon Stumpf, ${ }^{28} \mathrm{~d}$ 'avoir une vision d'ensemble d'un problème social avant de le subdiviser. Les causes des problèmes sociaux telles que définies sont complexes et sont reliées à des facteurs d'ordre individuel, familial, aux groupes, aux organisations et aux communautés. Cette interrelation des différents facteurs doit donc être reconnue non seulement au plan de la définition des problèmes mais aussi au niveau des moyens utilisés pour les réduire.

Il existe différentes orientations ou perspectives théoriques ayant permis le développement de diverses approches intégrées. Pour sa part, Garvin s'est attardé à comparer les différentes approches pour une pratique généraliste. Ceci l'a amené à une classification représentée sous trois vocables, selon les théories de base qui ont guidé les auteurs. II s'agit de la perspective centrée sur la tâche, de la perspective systémique et enfin, de la perspective psycho-sociale. ${ }^{29}$

De leur côté, Moreau, Lévesque et Panet-Raymond ${ }^{30}$ établissent une distinction entre les approches intégrées consensuelles et conflictuelles. Ils proposent une approche structurelle qui essaie de tenir compte des rapports inégalitaires qui existent entre les différents systèmes en présence dans une situation-problème. Cette approche accorde une grande importance à l'influence des facteurs structurels, telles les conditions de vie et de travail, sur les comportements personnels et interpersonnels. ${ }^{31}$ 
Les approches intégrées ont tenté d'élaborer un cadre théorique d'intervention qui exprime de façon satisfaisante la complexité des rapports sociaux qui constituent le champ d'analyse et d'intervention du service social.

Au plan de l'intervention, Alice Home ${ }^{32}$ qualifie ces efforts de méthodes "communautaires" puisque, dit-elle, elles sont davantage axées sur le social et elles requièrent l'accomplissement de rôles moins autoritaires et plus flexibles que ceux qui caractérisent les approches individualisées.

Selon elle, cette orientation communautaire remplace le modèle médical par un modèle de développement qui s'appuie davantage sur les ressources et les dynamismes des personnes et des communautés.

Cette perspective d'action exige, selon elle, que l'organisme qui distribue les services de même que les intervenants acceptent de confier plus de pouvoir aux personnes et aux collectivités.

\section{Présentation de différentes approches intégrées en service social 33}

Les modèles d'intervention analysés par Garvin fournissent une base théorique incitant à agir non seulement au niveau des effets des problèmes sociaux mais aussi au niveau de leurs causes. Cette perspective globale nécessite donc l'utilisation d'une grande variété de méthodes d'intervention.

Ainsi, les théoriciens ayant développé la perspective centrée sur la tâche voient les problèmes sociaux comme des tâches que les personnes doivent accomplir. L'aspect auquel les travailleurs sociaux s'intéressent est présenté comme étant les efforts faits par les gens pour accomplir ces tâches. ${ }^{34}$

Le concept implique également des possibilités de développement et de crises. ${ }^{35}$ L'intervention doit viser la croissance ou le développement des personnes mais également celui de son environnement. L'attention se porte ainsi sur les phénomènes d'adaptation auxquels les personnes sont soumises de même que sur la modification des demandes de l'environnement.

Les théoriciens de la perspective systémique conçoivent la personne en difficulté comme étant un système social en interaction avec différents systèmes : famille, école, travail, amis, institution qui distribue les services sociaux, etc. La cible de l'intervention est présentée comme une aide qu'il faut apporter aux différents systèmes afin de leur permettre d'interagir de façon à ce que les besoins de chacun soient respectés. 
Les tenants de la perspective psycho-sociale, quant à eux, précisent qu'une situation peut être fonctionnelle ou dysfonctionnelle. Une situation est définie comme fonctionnelle lorsqu'elle permet de répondre à la fois aux besoins des individus et des systèmes auxquels ils sont reliés.

\section{Le modèle centré sur la tâche}

Studt ${ }^{36}$ affirme qu'il est nécessaire, dans une première étape, d'analyser un problème en prenant en considération l'ensemble des facteurs qui interviennent. Le choix du niveau d'intervention se fait, dans une deuxième étape, et découle d'une analyse multidimensionnelle. L'auteur affirme que la tâche à accomplir est l'élément qui doit déterminer la méthode: action auprès de la personne, intégration à un groupe d'entraide ou action auprès des institutions ou des structures sociales.

De plus, le choix des objectifs et des tâches à accomplir doit découler d'une entente ou d'un accord explicite entre la ou les personnes concernées et l'intervenant. On effectue ainsi une distinction entre une aide apportée à une personne et l'imposition des idées et des valeurs d'un expert à un client. "On met à juste titre l'accent sur la nature secondaire et indirecte de l'aide, apportant ainsi un correctif à la tendance naturelle de l'expert à percevoir ses propres idées comme plus réalistes et mieux pensées que celles de son client. „ 37

Dans cette perspective, l'institution qui dispense les services sociaux cesse d'être un endroit où des professionnels sont chargés de "faire quelque chose" pour des clients plus ou moins passifs. Elle devient un lieu où des personnes, exerçant différentes fonctions, travaillent en collaboration avec les populations afin d'atteindre les objectifs souhaités par celles-ci.

Selon Studt, ${ }^{38}$ la participation active des populations et des intervenants implique une modification du modèle d'organisation du travail. Le modèle bureaucratique ou médical devrait céder la place à une série de modèles beaucoup plus souples, conçus dans le but de faciliter l'accomplissement des tâches.

Les principales caractéristiques de ce modèle devraient, selon Studt, favoriser différentes modalités d'intervention, permettre une utilisation flexible et créatrice des ressources humaines, encourager une délégation de l'autorité et des responsabilités, et faciliter la communication entre les personnes qui exercent différentes fonctions.

Concrètement, Studt préconise la formation d'une équipe de travail formée de représentants des usagers qui sont vus comme des partenaires-clés, de travailleurs sociaux, de chercheurs, d'administrateurs et du personnel clérical. Cette équipe se voit confier la responsabilité 
de l'élaboration, de la gestion et de l'évaluation des programmes destinés à la communauté.

Dans cette optique, les travailleurs sociaux participent à la planification des services en se basant sur les connaissances qu'ils possèdent des personnes et des populations. Ils doivent ainsi partager leurs connaissances avec les autres membres de l'équipe occupant des fonctions différentes. En conséquence, certains rôles de gestion réservés jusque-là à l'administration se trouvent délégués à l'équipe.

Cette organisation du travail permet selon Studt un meilleur partage de l'autorité en plus de favoriser une plus grande implication des administrateurs vis-à-vis la communauté. L'administration, affirme-t-il, se sent directement responsable des besoins des personnes et perçoit davantage l'impact de ses décisions sur leur vie.

Au plan des actions concrètes, la première étape consiste à se mettre à l'écoute des besoins tels qu'identifiés par les usagers. Une fois cette étape franchie, différentes méthodes peuvent être utilisées afin de permettre l'atteinte des objectifs : intervention individualisée, travail auprès de groupes, activités de recherche, activités politiques visant à mobiliser l'opinion publique, conférences, activités administratives dans le but de redéfinir les rôles, modifier les politiques de l'institution, mettre sur pied un comité d'usagers, etc.

Le modèle proposé par Studt met en évidence la nécessaire participation des personnes et des communautés à l'identification de leurs besoins. Elle insiste, en outre, sur l'importance d'utiliser l'expertise développée par les travailleurs sociaux dans le processus de planification des services. Le concept d'équipe multidisciplinaire est vu comme un moyen privilégié permettant l'atteinte de ces objectifs.

Les modèles qui s'inspirent de la théorie systémique ont, pour leur part, cherché à rompre avec une vision monolithique des phénomènes sociaux en mettant en évidence l'interrelation qui les caractérise. Cette approche globale, comme nous le verrons dans la section qui suit, se traduit par un élargissement de la nature des activités permettant de réaliser la croissance des personnes mais aussi celle des systèmes auxquels elles appartiennent.

\section{Le modèle systémique}

Le modèle systémique offre une représentation de la réalité sociale dans laquelle la personne et son environnement forment les parties d'un système qui interagissent et s'influencent mutuellement. La croissance ou le développement des systèmes se trouvent ainsi reliés à la nature des échanges ou des interactions qui s'établissent. 
Hearn affirme que la cible de l'intervention a été concentrée, à une époque, du côté de la personne en s'appuyant sur les théories psychologiques et, à une autre époque, du côté de l'environnement en s'inspirant des théories sociologiques et économiques. Or, une telle approche ne traduit pas la complexité et la dynamique des échanges qui existent entre la personne et son environnement. Dans cette perspective, la cible de l'intervention ne devrait pas être axée sur un système personne ou environnement - comme formant une entité distincte mais plutôt sur les relations entre les systèmes.

"Le focus central du service social se situe à l'interface ou au point de rencontre de la personne et de l'environnement - au point où il y a harmonie ou non, avec toutes les bonnes et mauvaises conséquences pour la personne et l'environnement. Le phénomène impliqué à cette interface est la transaction entre la personne et l'environnement. La transaction, c'est un échange dans un contexte d'action ou d'activité. Cette action ou cette activité est un mélange qui implique une personneactivité qui se confronte à un environnement-activité. " 39

Ce déplacement théorique implique une nouvelle façon de voir la personne en situation. L'évaluation d'une situation problématique doit ainsi accorder une place importante aux facteurs de l'environnement au lieu de s'intéresser principalement aux aspects intrapsychiques de la vie des personnes.

Cette perspective constitue un effort pour élaborer un cadre théorique qui permet de surmonter la dichotomie psychothérapie ou changement social, dichotomie qui reflète une vision fragmentée de la réalité sociale.

Cette vision globale se veut plus réaliste et plus appropriée que l'approche axée sur la division du travail auprès des personnes, des groupes ou des communautés. Selon Stumpf, l'approche traditionnelle fractionne le problème avant d'avoir obtenu au préalable une vision d'ensemble de la situation. Les modalités d'intervention sont, en effet, déterminées à partir de la fonction occupée dans l'organisation.

L'approche par méthodes séparées nous a conduits à fractionner un problème, avant de le voir comme un tout. Il favorise une prédétermination de la localisation du point d'entrée stratégique, sur la base de ce que le travailleur social est payé pour faire, soit de voir des clients individuellement ou en groupe, d'orienter le développement des politiques et de planifier des programmes. » 40

Cette vision globale devrait se traduire, au plan de l'action, par un ensemble d'activités s'adressant aux personnes et aux collectivités. Ces activités devraient également prendre en considération le contexte 
organisationnel afin de modifier les pratiques susceptibles de nuire à la qualité de services.

Pincus et Minahan ${ }^{41}$ proposent une approche intégrée dont l'objectif est centré sur les interactions entre les personnes et leurs systèmes ou réseaux de ressources. Ceux-ci constituent des systèmes de ressources informels (familles, amis, etc.), formels (syndicats, groupes de pression) et des systèmes de ressources sociales (hôpitaux, écoles, lieux de travail). Les personnes sont perçues comme dépendantes de ces différents systèmes qui leur procurent des ressources matérielles, émotives, spirituelles leur permettant de réaliser leurs aspirations ou leurs tâches.

Le cadre de référence de l'intervention sociale proposé par Pincus et Minahan comporte trois dimensions. La première reprend l'idée que les personnes doivent accomplir différentes tâches au cours de leur vie alors que la deuxième met en évidence les interactions qui existent entre les personnes et leurs réseaux de ressources. Le troisième aspect se rapporte à la relation entre les problèmes personnels des gens et leurs conséquences sociales. Le concept de tâche se rapporte aux exigences auxquelles les personnes sont soumises dans leur vie quotidienne : faire partie d'une famille, fréquenter l'école, faire son entrée sur le marché du travail, se marier, élever des enfants. II recouvre également les événements traumatisants tels la maladie, les séparations, les deuils, les difficultés financières auxquels les personnes sont confrontées. Ces événements sont décrits comme des tâches requérant des attitudes ou des actions de la part des personnes impliquées dans la situation.

La deuxième dimension de l'approche consiste à prendre comme cible l'interaction entre les personnes et leur réseau de ressources. Ceci implique que les problèmes ne sont pas perçus comme une caractéristique des personnes mais plutôt comme une caractéristique de leur situation sociale.

"La question n'est pas qui a le problème, mais comment les éléments d'une situation (incluant les caractéristiques des personnes impliquées) interagissent pour empêcher les personnes de venir à bout de leurs tâches. ॥ ${ }^{42}$

Les auteurs montrent qu'un problème de fonctionnement scolaire, par exemple, nécessite plusieurs interventions afin d'aider l'enfant, la famille et l'école à améliorer leurs relations. L'intervenant doit ainsi prendre comme cibles d'intervention l'enfant et la famille mais aussi les conditions à l'intérieur du milieu scolaire qui provoquent les problèmes.

Les auteurs établissent une distinction entre le bénéficiaire (immédiat ou ultime) de l'intervention et la cible de l'intervention. Le fait de considérer une personne comme bénéficiaire n'implique pas, selon 
Pincus et Minahan, qu'elle sera nécessairement la cible de l'intervention. Le bénéficiaire de l'intervention peut être une personne, un groupe ou une structure en faveur de qui l'intervention est effectuée et qui, à court terme ou à long terme, devrait en retirer les avantages.

La cible de l'intervention est la personne, le groupe ou la structure qui sera mobilisé par l'intervenant afin d'atteindre les objectifs fixés pour le bénéficiaire.

De cette distinction découle certaines actions en faveur d'une personne mais sans la participation de celle-ci. Ainsi, par exemple, une action au niveau de l'environnement scolaire n'implique pas nécessairement la ou les personnes qui en seront les bénéficiaires. Il en est de même d'actions visant la création de ressources communautaires. De plus, l'institution pour laquelle travaille l'intervenant peut devenir une cible d'intervention dans le but d'améliorer la qualité des services offerts.

Le troisième aspect de l'approche de Pincus et Minahan consiste, en effet, à inciter les intervenants à modifier le fonctionnement inadéquat des institutions:

«...l'intervenant peut ou non avoir des responsabilités administratives formelles dans son propre système sociétal, mais il est responsable d'essayer de changer un fonctionnement défectueux de son système, qui empêche celui-ci de fonctionner dans les meilleurs intérêts de ses bénéficiaires. " 43

Les intervenants devraient, par exemple, attirer l'attention sur l'absence de certaines ressources et sur les aspects dysfonctionnels des politiques et des législations sociales. Ils devraient également travailler à la mise sur pied de nouveaux services de même qu'à la coordination et à l'intégration des réseaux de ressources.

Dans le but d'influencer les politiques sociales, des informations peuvent être recueillies dans les dossiers de l'institution et des enquêtes peuvent être menées sur un problème précis. De plus, les intervenants peuvent exercer des pressions sur leur institution dans le but de prendre position face à certains problèmes. Les intervenants peuvent également entreprendre différentes actions externes, comme la formation d'un comité de citoyens, afin de solutionner certains problèmes sociaux.

\section{La perspective psycho-sociale}

La troisième perspective identifiée par Garvin est celle qui utilise les concepts développés en sciences sociales. 
Elle s'appuie sur des recherches portant sur les rôles, les structures des petits groupes et les ressources d'influence sociale. L'influence de la modification du comportement telle que développée en service social clinique et de la théorie de l'échange en sociologie appartiennent à ce courant théorique. Garvin et Glasser, par exemple, utilisent la dynamique des petits groupes que l'on peut observer dans la dyade, la famille, et les situations de groupe. L'intervenant est alors défini comme un expert qui utilise cette information afin de provoquer un changement avec ou en faveur du client. ${ }^{44}$

Siporin s'appuie, pour sa part, sur les concepts de la théorie des rôles et de la théorie des groupes. Il affirme qu'une situation sociale fait référence à un groupe social en action, à un moment donné et dont l'intérêt est centré vers un objet, une personne ou un but significatif. Le champ d'analyse est principalement les situations où se retrouvent des petits groupes, bien que Siporin affirme s'intéresser également aux groupes plus importants tels les foules et les mouvements sociaux. ${ }^{45}$ Les techniciens psycho-sociaux utilisent les concepts systémiques mais ce sont surtout les unités primaires et plus petites qui font l'objet de leur attention.

La cible de l'intervention, dans la perspective psycho-sociale, se rapproche de celle identifiée dans la perspective centrée sur la tâche puisqu'elle est définie comme étant le fonctionnement social.

Siporin précise cependant qu'une situation peut être fonctionnelle ou dysfonctionnelle. Selon lui, une situation sociale est fonctionnelle lorsqu'elle permet de répondre aux besoins à la fois des individus et des systèmes sociaux. Les concepts psycho-sociaux se rapportant aux petits groupes peuvent ainsi s'appliquer au fonctionnement individuel des clients ou aux concepts sociologiques de situation tels que développés par Siporin.

Dans la perspective psycho-sociale, on retrouve l'idée de conclure une entente avec le client. L'intervenant se définit comme un expert qui choisit le ou les systèmes d'action appropriés (individus, familles, groupes) et utilise des moyens directs afin d'atteindre les objectifs fixés au moment de l'entente.

Les auteurs s'appuient sur différentes théories permettant d'accomplir diverses tâches: psychologie du moi, modification de comportement, théories des rôles et des petits groupes, théorie des organisations.

Garvin ${ }^{46}$ a effectué une analyse comparative des trois modalités d'approches intégrées à partir de cinq dimensions: la cible de l'intervention (problèmes et phénomènes qui constituent le champ d'action du service social), le rôle du travailleur social, les bases de connaissances, 
les composantes au répertoire d'intervention professionnelle, et les conséquences que suggère chacune des orientations sur la formation.

Nous analyserons les quatre premières dimensions afin de mieux saisir les principales caractéristiques qui se dégagent de ces différentes orientations. Quant à la cinquième dimension, elle n'a pas été retenue dans l'étude de Charlevoix portant essentiellement sur un modèle de pratique.

\section{Analyse comparative de trois approches intégrées ${ }^{47}$}

\section{La cible de l'intervention}

\section{Le modèle centré sur la tâche}

Pour Bartlett, la cible de l'intervention est le fonctionnement social, c'est-à-dire l'amélioration de la croissance du client résultant de l'effort effectué dans l'accomplissement de ses tâches:

«Bartlett (...) voit le développement de la croissance du client, provoqué par ses efforts pour accomplir ses tâches, comme le focus du travail social et elle explique ce processus à l'intérieur de sa définition du concept de fonctionnement. ${ }^{48}$

Selon l'auteure, la croissance est possible lorsqu'il existe un équilibre entre les efforts faits par les personnes et les pressions de l'environnement.

L'attention de l'intervenant est centrée sur les phénomènes d'adaptation tels qu'exprimés dans la psychologie du « moi » et sur la modification des demandes de l'environnement.

\section{Le modèle systémique}

Les théoriciens qui s'inspirent de l'approche systémique ne voient pas le client comme une personne qui essaie de s'adapter mais plutôt comme un système social qui interagit avec d'autres systèmes et qui effectue une entente avec un système agent de changement.

L'intervention consiste à aider les différents systèmes en présence à agir de façon à permettre l'atteinte des buts ou des objectifs de chacun des systèmes. 
"Alors, le focus du travail social a tendance à être vu comme d'aider les systèmes en présence à intéragir de façon à maximiser l'atteinte des buts de chacun des systèmes. ${ }^{49}$

Dans cette perspective, le fonctionnement de l'individu mais aussi celui des systèmes sociaux est la cible de l'intervention.

\section{La perspective psycho-sociale}

Garvin et Glasser affirment que le fonctionnement social est la cible de l'intervention. Siporin précise cependant qu'une situation peut être fonctionnelle ou dysfonctionnelle. Selon lui, une situation sociale est fonctionnelle lorsqu'elle permet de répondre aux besoins à la fois des individus et aussi des systèmes sociaux. Les concepts psycho-sociaux se rapportant aux petits groupes s'appliquent ainsi au fonctionnement individuel ou aux concepts sociologiques de situation tels que développés par Siporin.

\section{Les tâches du travailleur social}

Les tâches effectuées par le travailleur social constituent la deuxième dimension analysée par Garvin. Elle concernent a) l'étendue des activités accomplies: le travailleur social intervient-il auprès d'un système ou auprès de plusieurs? b) quelles sont les attentes de rôles auxquelles l'intervenant est confronté?

\section{Le modèle centré sur la tâche}

Bartlett montre que l'action du travailleur social se caractérise par un processus d'intervention comportant différentes phases: dans la phase initiale, le travailleur social perçoit ou analyse les situations sociales, en prenant en considération les efforts d'adaptation faits par les personnes pour solutionner leurs problèmes en réponse aux pressions de l'environnement, et les conséquences pour les deux s'il existe un déséquilibre. Le travailleur social effectue ensuite sa propre évaluation de la situation et utilise un répertoire d'intervention. Ce répertoire appartient à la profession et non à une méthode en particulier. Bartlett affirme cependant que certains seront davantage portés vers les services aux personnes tandis que d'autres choisiront de changer l'environnement. 
"(...) certains vont insister sur le fait de donner des services directs aux personnes aux prises avec des problèmes, et d'autres vont accorder plus d'importance aux changements dans l'environnement social (...). ${ }^{50}$

D'après elle, cependant, le répertoire s'agrandit au fur et à mesure qu'un travailleur social devient plus expérimenté.

Les interventions - évaluation des situations et planification des interventions - ne sont plus compartimentées par méhode. Elles sont plutôt une base commune qui s'applique à l'ensemble de la profession. Bartlett, Studt et Siporin affirment que la situation est le lieu de l'intervention.

\section{Le modèle systémique}

Les auteurs ne s'entendent pas sur le rôle que devrait jouer le travailleur social. Pincus et Minahan affirment que l'utilisation de la théorie des systèmes peut servir d'instrument d'analyse et de planification, quel que soit le rôle joué par le travailleur social. D'autres auteurs précisent cependant que les activités peuvent s'adresser à un système précis, c'est-à-dire, la personne ou l'environnement, ou encore à la relation entre les systèmes.

L'approche de Hearn montre, pour sa part, que le travailleur social fait partie des situations sociales. Il se situe à la frontière des systèmes, entre les individus et les situations. Les processus qui surviennent entre les systèmes sont affectés par les entrées ("inputs") et les sorties (" outputs ") venant des autres systèmes. Schwartz, ${ }^{51}$ pour sa part affirme que le rôle consiste à médiatiser et faciliter les transactions systémiques. Selon lui, l'évaluation des dynamiques internes et la planification des objectifs peuvent nuire à la médiation entre les systèmes.

\section{La perspective psycho-sociale}

Garvin et Glasser ont formulé des concepts qui s'appliquent à l'intégration de l'approche individualisée et de groupe. Ils identifient des étapes d'intervention telles l'évaluation et l'intervention.

Le comportement dysfonctionnel du client peut être une cible d'intervention. On cherche à en venir à une entente ou à un contrat avec le client, et le travailleur social joue le rôle d'un expert en choisissant le ou les systèmes d'action appropriés (individus, famille ou groupe) et en utilisant des moyens directs et indirects pour atteindre les 
objectifs fixés. Les théoriciens s'appuient sur les sciences sociales et les sciences du comportement afin de proposer des procédures de changement. En résumé, dans le modèle axé sur la tâche, l'intervenant est un facilitateur qui veut promouvoir la croissance ; dans le modèle systémique, il est un médiateur et dans le modèle psycho-social, il est un expert du changement.

\section{Les bases de connaissances}

Garvin affirme que les modèles analysés ne présentent pas l'ensemble des informations scientifiques disponibles en service social. Chacun s'appuie sur des concepts et des théories particulières. Ainsi, l'approche centrée sur la tâche s'inspire de la théorie du "moi». Cette théorie s'intéresse au comportement des individus en situation de stress, à la signification qu'ils attribuent à la situation, aux efforts qu'ils font pour s'adapter, aux façons d'utiliser l'aide disponible.

L'approche systémique utilise les concepts et la théorie générale des systèmes. Le comportement est perçu comme une conséquence de l'ensemble d'une situation dans laquelle se retrouvent un sous-système individuel, un sous-système de groupe et d'autres unités sociales. Les différents systèmes se maintiennent dans le temps mais les unités sociales qui les composent peuvent changer.

L'approche psycho-sociale définit plus ou moins le genre de transactions auxquelles doit s'intéresser le travailleur social. Elle s'appuie plutôt sur différentes théories pour accomplir différentes tâches : psychologie du "moi ", modification de comportement, théorie des rôles, théorie des petits groupes et théorie des organisations.

\section{Les composantes du répertoire d'action professionnelle}

Le répertoire d'action peut être décrit comme étant les activités que l'intervenant effectue en essayant de provoquer un changement chez le client ou dans sa situation. Le modèle centré sur la tâche se préoccupe surtout du répertoire de la profession et moins du répertoire du travailleur social. Il suggère l'idée d'une équipe professionnelle qui planifie et gère les activités :

[L'idée d'une équipe de professionnels va de soi ici, et l'approche de Studt montre des membres du personnel qui planifient et dirigent "le programme communautaire pour la prise en charge totale des cas de l'agence. 》] ${ }^{52}$. 
Bartlett entrevoit des problèmes lorsqu'aux activités d'intervention individualisée et de groupe viendront s'ajouter celles d'organisation communautaire et d'élaboration de politiques. Elle se demande comment ces dernières interventions vont pouvoir s'intégrer dans un cadre théorique de fonctionnement social ayant ses propres valeurs.

Dans le modèle systémique, les actions viseront à augmenter la communication, la rétroaction et la négociation. L'accent est mis sur les habiletés de résolution de problème en relation au fonctionnement systémique et à l'échange entre les systèmes.

Dans le modèle psycho-social, chaque orientation théorique propose des interventions particulières.

Garvin affirme que le modèle psycho-social vient clarifier les interventions, mais il n'exprime pas suffisamment les valeurs de la profession en ce qui concerne le choix des interventions. Les modèles systémiques sont pertinents quant au choix des interventions à effectuer. Quant au modèle axé sur la tâche, il met en évidence l'importance des valeurs dans le choix des interventions de même que leur pertinence pour les tâches à accomplir.

L'auteur conclut en soulignant les aspects positifs et négatifs des différents modèles. Ainsi, l'approche centrée sur la tâche suggère, selon lui, l'importance des buts et des activités spécifiques. Par contre, si elle offre une multitude de concepts sur les transactions personnesituation, elle en propose très peu sur les systèmes plus vastes.

Le modèle systémique permet de solutionner ce problème. Il peut être utile pour effectuer un choix des cibles d'intervention. II n'est pas suffisamment précis, cependant, concernant le processus plus immédiat et les activités de changement. Le modèle psycho-social en offrant les instruments de changement social vient combler les besoins de techniques et de stratégies. Il devrait, cependant, affirme Garvin, inclure les définitions des tâches professionnelles et des considérations au niveau du macrosystème.

Les limites de chacun des modèles peuvent être surmontées, selon l'auteur, en les combinant.

Des théoriciens québécois ont élaboré un modèle d'intervention intégré qui postule clairement une distribution inégale du pouvoir. En conséquence, les intervenants et intervenantes doivent prendre partie en faveur des personnes, des groupes ou des collectivités qui se voient privés d'un tel pouvoir.

L'approche développée par Moreau, Lévesque et Panet-Raymond que nous analyserons dans la section suivante propose une version radicale de l'intégration. Tout en s'inspirant des approches "consensuelles", elle représente une tentative d'intégrer les aspects 
conflictuels qu'elle juge avoir été laissés de côté par Pincus et Minahan et certains autres théoriciens.

\section{Le modèle structurel}

Ce modèle s'appuie sur l'hypothèse que les conditions matérielles. d'existence jouent un rôle déterminant sur la vie des personnes et des collectivités.

"Dépendamment de la position dans l'organisation et la division du travail, selon l'âge, le sexe et la classe sociale, les conditions de vie et de travail sont en grande partie déterminantes de la santé physique et de la façon de penser, de sentir, d'agir des individus et des collectivités. " 53

Ainsi donc, les auteurs considèrent que "les rapports entre les systèmes sont antagonistes, opposés et inégalitaires. "

Par ailleurs, la personne est vue comme pouvant contribuer par ses attitudes et comportements à la situation-problème. L'intervenant devra alors jouer un rôle de conscientisation :

"On se souciera donc de changer les comportements, les idées et les sentiments vécus par la cliente, là où ceux-ci maintiennent, supportent et légitimisent la situation d'oppression dans laquelle elle vit. " 54

"L'action vise à désindividualiser et à collectiviser les problèmes vécus comme individuels. " 55

Suite à cette première intervention, les auteurs de cette approche préconisent une démarche de conscientisation qui doit permettre à leurs clients de "faire ressortir les aspects socio-politico-économiques de la situation et favoriser le développement d'actions collectives pour corriger cet état de choses. $" 56$

Ainsi, au plan théorique, l'analyse d'une situation problème doit tenir compte de l'ensemble des facteurs susceptibles d'intervenir. On prendra donc en considération les facteurs d'ordre psychologique, biologique, idéologique, culturel, social, économique et politique.

Au plan de l'intervention, l'action évolue selon un continuum, ou s'effectue parallèlement à différents niveaux, selon la nature des difficultés. D'après les auteurs, il est important de considérer tous les niveaux possibles de l'intervention. À partir de la situation-problème, l'action sera centrée au niveau personnel/interpersonnel ou encore institutionnel/communautaire. Ainsi, un support individualisé pourra aider les personnes à surmonter certains obstacles d'ordre psychologique ou interactionnel qui les empêchent de réaliser leurs objectifs. 
Toutefois, il importe de " toujours comprendre les effets des conditions matérielles - de travail et de vie - sur les comportements personnels et interpersonnels et de toujours intervenir pour changer ces conditions avant d'intervenir aux niveaux personnels et interpersonnels. „ ${ }^{57}$

Un deuxième niveau d'action permet pour sa part, l'amorce d'une réflexion critique entre les individus. La démarche retenue à ce niveau permet de regrouper les gens concernés par une même situationproblème ou de les référer à des groupes déjà existants dans un but d'entraide et de les orienter vers une action collective.

L'intervention collective favorise particulièrement deux types d'action : "la mise sur pied de ressources et de services d'entraide mutuelle afin de soulager de façon immédiate les tensions et le développement d'une base de pouvoir pouvant servir à la revendication à plus long terme de réformes. ${ }^{58}$

L'institution qui distribue les services représente un autre niveau d'action. Comme dans l'approche systémique, elle peut devenir une cible d'intervention puisque, affirment les auteurs, elle n'est pas exempte de conflits de classes. Les intérêts de l'institution, des intervenants et des clientèles sont susceptibles d'entrer en contradiction. II devient alors nécessaire d'entreprendre des actions dont le but est de défendre les droits des personnes, de négocier en leur faveur ou de les référer à d'autres organismes.

Dans cette perspective, les auteurs préconisent la mise sur pied de structures permettant aux clientèles d'exercer un contrôle réel sur l'organisme qui dispense les services.

Dans le but de dépasser les contraintes inhérentes au contexte de travail dans une institution publique, Moreau, Lévesque et PanetRaymond suggèrent aux intervenants de prolonger les actions entreprises à l'interne en joignant différents mouvements sociaux. Ainsi, des liens peuvent s'établir avec les syndicats, les mouvements féministes, écologiques ou autres afin de transformer radicalement les structures sociales, politiques et économiques qui sont oppressantes.

\section{Les limites de l'approche intégrée}

Malgré l'intérêt que l'on puisse porter à l'approche intégrée, que l'on soit intervenant ou bénéficiaire d'une intervention de ce type, quelques auteurs ont relevé un certain nombre d'interrogations portant sur des éléments bien précis tels : le pouvoir et l'autorité du travailleur social, l'état des connaissances en travail social et le développement du travail par équipe. 
En effet, Home fait remarquer que la perspective d'avoir à partager le pouvoir fait peur aux intervenants et aux organismes. Les intervenants, affirme-t-elle, éprouvent de la difficulté à délaisser leur rôle d'expert pour accorder une plus grande autonomie aux communautés.

\footnotetext{
"Tout comme ceux qui travaillent avec de petits groupes, les nouveaux praticiens en intervention collective éprouvent de la difficulté à faire confiance aux collectivités et à savoir quand les aider sans s'imposer à elles. " 59
}

Par ailleurs, cette orientation est perçue comme menaçante par les organismes de distribution de services parce qu'elle amène une prise de conscience vis-à-vis les causes sociales des situations-problèmes et suscite des revendications de la part des usagers. Pour ces raisons, le travail auprès de groupes ou de collectivités est considéré "comme un luxe ou un divertissement qui enlève des énergies aux services individualisés. " 60

Alice Home ${ }^{61}$ affirme aussi que l'intervention de groupe et l'intervention collective amènent des résistances de la part de l'organisme qui se demande, entre autres, si les actions entreprises sont conformes au mandat d'aide psycho-sociale qui lui est confié. Elles posent, également, le problème de la comptabilisation des activités qu'il devient difficile à évaluer selon les normes statistiques existantes.

Une autre interrogation soulevée concerne les perspectives d'intégration faisant l'hypothèse qu'une seule personne peut assumer l'ensemble des tâches découlant d'une évaluation multidimensionnelle. Les perspectives systémique et structurelle jugent qu'une même personne peut intervenir auprès des individus, des groupes, des collectivités, des organisations et contribuer à modifier les législations sociales. Certains auteurs remettent en question cette position. Selon eux, une personne ne peut développer une compétence la rendant apte à intervenir efficacement à tous les niveaux (Specht, ${ }^{62}$ Studt, ${ }^{63}$ Evans ${ }^{64}$ ). Il y aurait lieu sans doute de prendre en considération différentes variables afin d'être en mesure de porter un jugement valable sur cette affirmation. Comme le suggère Bartlett, le nombre d'années d'expérience exerce peut-être une influence positive en ce sens qu'un travailleur social plus expérimenté peut élargir son répertoire d'intervention. Le seul facteur expérience pris isolément ne nous autorise cependant pas à formuler une hypothèse aussi optimiste. Il faudrait plutôt prendre en considération d'autres variables telles l'intérêt et la motivation de l'intervenant à travailler dans une perspective généraliste plutôt que spécialisée.

La solution mise de l'avant consiste à développer le travail d'équipe. Si les travailleurs sociaux évaluent l'ensemble des facteurs qui interviennent dans une situation-problème, on ne peut s'attendre à ce qu'une 
seule personne effectue toutes les tâches. Il faudra alors envisager les implications au plan des méthodes de travail et des structures organisationnelles.

Une autre difficulté soulignée par Evans concerne la base de pouvoir ou d'autorité de l'intervenant. Au nom de quelle autorité le travailleur social agira-t-il? Au nom de l'organisation qui l'emploie ou en se référant à des valeurs d'ordre professionnel ? ${ }^{65}$ De plus, la définition des problèmes étant élargie, elle risque de dépasser la fonction attribuée à l'organisation. Quelles sont alors les conséquences sur la relation entre les travailleurs sociaux et leur employeur? Quel type d'organisation peut accepter les actions visant un changement social et une action au niveau des politiques sociales? Evans se demande si de telles exigences vont augmenter les zones possibles de conflits employeurs-employés.

\section{Notes et références}

1 René AuCLAIR et Christiane LAMPRON, Approche intégrée: une innovation dans la dispensation des services sociaux, Recherche subventionnée par le Conseil québécois de la recherche sociale (C.Q.R.S., no ISBN 2-920755-05-6), Québec, 1987 : 27-63.

2 Note méthodologique :

Afin de cerner la documentation existante sur l'approche intégrée en service social, l'équipe de recherche a privilégié une recherche documentaire visant à l'exhaustivité.

Le repérage de la littérature existante s'est effectué rétrospectivement depuis le début des années 65 , à partir de plusieurs bassins documentaires : service social et sciences sociales en général. Dans chacun de ces domaines, plusieurs sources d'informations spécialisées ont été dépouillées, notamment :

- Documentation en service social

1. Social Work Education : A Bibliography : cette bibliographie sur l'approche intégrée en service social couvre la période 1965-1984. La méthodologie suivie par le compilateur nous assure de la qualité et de l'exhaustivité de cet outil documentaire.

2. Social Work Research and Abstracts : cette bibliographie dépouillant régulièrement plus de 200 revues de service social a permis de couvrir la période 1965-1984. Elle met à jour la bibliographie précédente.

- Documentation en science sociales

1. Social Scisearch : cette base de données bibliographiques, multidisciplinaire en sciences sociales, recense environ un million d'articles tirés de $\mathbf{1 5 0 0}$ périodiques de sciences sociales. Cet instrument a fourni quelques références bibliographiques répondant aux critères de sélection. 
2. Dissertation Abstracts : cette source d'informations accessibles par ordinateur comprend l'ensemble des thèses doctorales canadiennes et américaines depuis 1861. À ce jour, plus de $\mathbf{8 0 0 0 0 0}$ thèses dans toutes les disciplines composent cette base de données. Suite à l'application de notre stratégie recherche, aucune thèse ne fut répertoriée.

Le dépouillement des outils documentaires imprimés et automatisés révèle certaines caractéristiques de la littérature appartenant à l'approche intégrée en service social.

On ne peut cependant parler d'une répartition selon la langue de ce corpus documentaire, car presque tous les documents répertoriés sont de langue anglaise. La très grande majorité des auteurs sont américains. Le peu d'articles en langue française incite les auteurs de ce rapport à vouloir combler ce vide. Cette constatation nous a donc amenés à développer :

- une bibliographie commentée des documents répertoriés (publication séparée) ;

— une revue de littérature. Cette analyse détaillée vise à dégager les orientations émanant d'une approche intégrée en service social.

3 Voir: Harry SPECHT; "Generalist and specialist approaches to practice and a new educational model ", dans : Frank Clark, Morton Arkava et al., The Pursuit of Competence in Social Work, San Francisco et Londres, Jossey-Bass, 1979, p. 2.

4 Alice HOME, "Le passage de l'intervention auprès des petits groupes et des collectivités ", Service social, vol. 29, n 1-2, janvier-juin 1980: 191-205.

5 Anatole SHAFFer, "Community organization and the oppressed", Education for Social Work, vol. 8, n 3, 1972: 67.

6 Une méthode peut être définie comme "un ensemble de techniques et de moyens concrets, appuyés sur des principes scientifiques et orientés vers un but de mieux-être [...] Par techniques, on entend les moyens détaillés [...] qui sont les éléments constitutifs de la méthode et qui lui permettent d'atteindre ses fins." Simone Pare, Groupes et service social, Québec, Les Presses de l'Université Laval, 1971, p. 3.

7 Bernard J. CouGHuIN, "Reconceptualizing the theoretical base of social work practice, dans : Lilian RIPPLE (éd.), Innovations in Teaching Social Work Practice, New York, Council on Social Work Education, 1970, p. 61.

8 Fédération des C.L.S.C. du Québec, Pour une approche communautaire des C.L.S.C. auprès des 0-18 ans, Direction de l'analyse et de l'évaluation des programmes, Montréal, 1982, p. 8.

9 Jack GEIGER, "Of the poor, by the poor, or for the poor: The mental health implications of social control of poverty programs, Psychiatric Research Reports, n० 21, 1967 : 55-71.

10 Miton S. Davis et Robert E. Tranquadea, "A sociological evaluation of the Watts neighboorhood health center", Medical Care, vol. 7, no 2, 1969: 105-117.

11 Daniel Mornihan, Maximum Feasible Misunderstanding, New York, Free Press, 1968.

12 Ibid. 
13 Karen TEIGISER, "Evaluation of education for generalist practice", Journal of Education for Social Work, vol. 19, no 1, 1983 : 79-85.

14 Charles GARVIN, "Education for generalist practice : a comparative analysis of current modalities ", dans: Teaching for Competence in the Delivery of Direct Services, New York, Council on Social Work Education, 1976 : 19-30.

15 Joseph ANDERSON, "Generic and generalist practice and the BSW Curriculum ", Journal of Education for Social Work, vol. 18, no 3, 1982: 37-45.

16 Allen Pincus et Anne Minahan, Social Work Practice : Model and Method, Itasca (III.), Peacock, 1974.

17 Ruth Middeman et Gale Goldberg, Social Service Delivery: A Structural Approach to Social Work Practice, New York, Columbia University Press, 1974.

18 Elliot STUDT, "Social work theory and implications for the practice of methods ", Social Work Education Reporter, vol. 16, no 2, 1968: 22-24 et 42-46.

19 Harry SPECHT, op. cit.

20 Bernard J. Coughuin, op. cit.

21 Louis LOWY, Leonard M. BLOKSBERG et Herbert J. WALBERG, Teaching Records: Integrative Leaming \& Teaching Project, New York, Council on social work Education, 1971 : VIII.

22 J.D. ANDERSON, op. cit., p. 44.

23 Ibid.

24 Charles Garvin, op. cit.

25 Allen Pincus et Anne Minahan, op. cit.

26 Charles Garvin, op. cit.

27 Roger Evans, "Some implications of an integrated model of social work for theory and practice ", British Journal of Social Work, vol. 6, n 2, 1976 : 177-200.

28 Jack STUMPF, "Teaching an integrated approach to social work practice", dans Lilian RIPPLE (éd.), Innovatons in Teaching Social Work Practice, New York, Council on Social Work Education, 1970 : 22-33.

29 Charles Garvin, op. cit.

30 Maurice Moreau, Justin Levesque et Jean PANET-RAYMOND, "L'approche structurelle: un défi pour les éducateurs en travail social», dans Justin LEVESQUE, Robert MAYER et al. La formation en travail social au Québec : d'une école à l'autre, Montréal, Regroupement des unités de formation universitaire du Québec, 1984 : 91-113.

31 Le modèle structurel, tel que présenté ici, est assimilé aux approches intégrées. Nous l'avons traité séparément aux fins de la recherche réalisée avec les praticiens du Centre des services sociaux de Québec à Charlevoix.

32 Alice HoMe, op. cit.

33 La partie portant sur la présentation des différentes approches intégrées est un résumé réalisé par madame Micheline $\mathrm{Cyr}$, chargée de recherche, du texte déjà cité de Charles Garvin.

34 Harriet M. BARTLET, The Common Base of Social Work Practice, New-York, National Association of Social Workers, 1970, cité par C. Garvin, op. cit., p. 19. 
35 William ReID et Laura EPSTEIN, Task-Centered Casework, New York, Columbia University Press, 1972, cité par C. GARVIN, op. cit., p. 19-30.

36 Elliot STUDT, op. cit.

37 Mathilde Du RANQUET, L'approche en service social, intervention auprès des personnes et des familles, Saint-Hyacinthe, Edisem et Paris, le Centurion, 1981, p. 203.

38 Elliot StUdT, op. cit.

39 Gordon Hearn, "Basic Constructs for an Integration and Generative Conception of Social Work", The General Systems Approach: Contributions toward an Holistic Conception of Social Work, New York, Council on Social Work Education, 1969, p. 7.

40 Jack StUMPF, op. cit., p. 26.

41 Allen Pincus et Anne Minahan, op. cit.

42 lbid., p. 11.

43 Ibid., p. 24.

44 Charles Garvin et Paul Glasser, "The Bases of Social Treatment", Social Work Practice, New York, Columbia University Press, 1970: 149-177, cité par C. Garvin, op. cit., p. 20.

45 Max SIPORIN, "Situational Assessment and Intervention ", Social Casework, vol. 53, no 2, 1972: 91-109, cité par C. GARVIN, op. cit., p. 21.

46 Charles Garvin, op. cit.

47 Francine Ouellet a utilisé l'analyse comparative des trois premières perspectives réalisée par Garvin pour en faire un tableau synthèse fort intéressant auquel nous avons ajouté les éléments de la perspective structurelle. L'auteure a fait ressortir les principaux éléments de chacune des trois premières approches sous différents vocables : but du service social; rôle principal du travailleur social; focus d'intervention; composantes d'une action professionnelle; théories de base ; auteurs de base ; implications en fonction du développement d'un curriculum. Voir Francine OUELLET, "Une conception de la formation et de l'évaluation en service social ", dans Justin LeVESQUE, Robert MAYER et al, op. cit., p. 75.

48 Charles Garvin, op. cit., p. 21.

49 Ibid., p. 22.

50 Harriett BARTLETt, The Common Base of Social Work Practice, p. 174, cité dans Charles Garvin, op. cit., p. 23.

51 William SChWARTZ, "The Social Worker in the Group ", Social Welfare Forum, Columbia University Press, 1967 : 146-177.

52 Charles Garvin, op. cit., p. 26.

53 Maurice Moreau, Justin Lévesque, Jean Panet-Raymond, op. cit., p. 92.

54 Ibid., p. 95.

55 Ibid., p. 105.

56 Ibid., p. 95.

57 Ibid., p. 93.

58 Ibid., p. 102. 
59 Alice Home, op. cit., p. 197.

60 Ibid., p. 195.

61 Ibid.

62 Harry SPECHT, op. cit.

63 Elliot StUdT, op. cit.

64 Roger Evans, op. cit.

65 Ibid. 